\title{
Preparation and evaluation of mucoadhesive hydrophilic hydroxy propyl methyl cellulose based extended release matrix tablets of Niacin (Nicotinic acid)
}

\author{
Md. Rezowanur Rahman, Sheikh Tasnim Jahan, Sams Mohammad Anowar Sadat and \\ Reza-ul Jalil
}

Department of Pharmaceutical Technology, Faculty of Pharmacy, University of Dhaka, Dhaka-1000, Bangladesh

\begin{abstract}
The aim of this study is to develop a once-daily extended release matrix tablet of Niacin using Hydroxy Propyl Methyl Cellulose i.e. Methocel K4M CR as release controlling factor and to evaluate drug release parameters as per various release kinetic models and also to find the best fitted extended release matrix formulation of Niacin. In order to achieve required extended release profile, tablets were prepared by wet granulation technique using different formulations (F-1, F-2, F-3, F-4, F-5 and F-6) with different amount of Methocel K4M CR. The formulated tablets were also characterized by physical and chemical parameters and results were found in acceptable limits. Different release kinetic models were applied in order to evaluate release of Niacin from the prepared matrix tablets. Criteria for selecting the most appropriate model were based on linearity (coefficient of correlation) and diffusion exponent of the respective kinetic model. Among all the formulations, F-5 was found as the best fitted matrix tablet for extended release of Niacin. Drug release mechanism was found as a complex mixture of diffusion, swelling and erosion.
\end{abstract}

Keywords: Niacin (Nicotinic Acid), Extended release, Methocel K4M CR, Wet granulation.

\section{INTRODUCTION}

In the mid 1950s, Niacin (Nicotinic Acid) was launched as the first effective lipid-modifying agent. Niacin reduces hepatic synthesis of triglycerides (TG) as well as the secretion of very low-density lipoprotein (VLDL) by inhibiting the mobilization of free fatty acids from peripheral tissues and also inhibits the conversion of VLDL into low-density lipoprotein (LDL). Niacin raises high-density lipoprotein (HDL) cholesterol by blocking hepatic uptake of apolipoprotein A-1, decreasing clearance, and increasing the amount of $\mathrm{HDL}$ cholesterol available for reverse cholesterol transport (Knopp et al., 1985; Grundy et al., 1981). Besides Niacin was the first lipid-altering agent shown to significantly reduce coronary death and nonfatal myocardial infarction, as well as total mortality, in patients with documented coronary heart disease in the Coronary Drug Project (CDP), a large, prospective trial (JAMA, 1975; Canner et al., 1986).

Niacin was made the first move as immediate release (IR) tablet and approved by US Food and Drug Administration (US FDA) and is available as over the counter (OTC) drug. But the primary limitation of IR or plain niacin is prostaglandin-mediated facial and truncal flushing, which is experienced by most patients during the initial days of treatment (Knopp, 2000). Although symptoms diminish over time, many patients stop therapy before tolerance develops. Other unwanted effects include itching, mucous membrane irritation, and diarrhea. Another limitation of niacin IR is the multiple-dosing requirement i.e. 2 or 3 times a day (Knopp, 1998). Sustained release (SR) Niacin tablet was developed in the 1960s to reduce flushing. But the clinical use of these products suggested an increased incidence of hepatotoxicity and gastrointestinal intolerance (McKenney et al., 1994).

To overcome unwanted effects of both IR and SR delivery system, extended release (ER) Niacin formulation has been introduced recently. Niacin ER has a delivery system allowing absorption rates intermediate to that of niacin IR and SR. These formulations were developed in an attempt to achieve the lipid-lowering efficacy of Niacin IR with a reduced incidence of flushing and to minimize the hepatotoxicity seen with Niacin SR. In this regard, mucoadhesive hydroxy propyl methyl cellulose 
polymer (Methocel K4M CR) was used to prepare Niacin ER matrix tablet formulations in various ratios.

\section{MATERIALS AND METHODS}

Niacin (Nicotinic acid) was a gift from Western Drugs Pvt. Ltd, India, Hydroxy Propyl Methyl Cellulose (Methocel K4M CR) (Colorcon Asia Pvt. Ltd., Bangladesh), Microcrystalline Cellulose (Avicel $\mathrm{PH}$ 101) (Veer Pharma Chem, Ahmedabad, India), Lactose (fine) (Mahaan Proteins Limited, New Delhi, India) were obtained and used as received. Materials and excipients used in preparing tablets were of Pharmaceutical grades. All other chemicals used were of analytical grade.
Preparation of matrix tablet: Matrix tablets (each tablet contains $500 \mathrm{mg}$ of niacin) were prepared by wet granulation technique.

The drug polymer ratio was developed to adjust drug release as per release profile of formulated ER tablets of Niacin in $900 \mathrm{ml}$ of purified water which is used as dissolution medium. Total weight of each tablet was kept constant for all the fabricated batches under experimental conditions of preparations. The composition of tablets of each formula in milligram is shown in Table 1.

Table 1: Composition of different formulations $(\mathrm{mg})$ of Niacin ER matrix tablets

\begin{tabular}{|l|c|c|c|c|c|c|}
\hline \multirow{2}{*}{ Ingredients } & \multicolumn{5}{|c|}{ Formulations } \\
\cline { 2 - 7 } & F-1 & F-2 & F-3 & F-4 & F-5 & F-6 \\
\hline Niacin & 500 & 500 & 500 & 500 & 500 & 500 \\
\hline Methocel K4M CR & 72 & 112 & 45 & 120 & 120 & 138 \\
\hline Lactose (fine) & 137 & 137 & 137 & 97 & 82 & 82 \\
\hline Microcrystalline Cellulose (Avicel PH 101) & 55 & 15 & 82 & 47 & 62 & 44 \\
\hline Magnesium Stearate & 6 & 6 & 6 & 6 & 6 & 6 \\
\hline Total Weight/Tablet & 770 & 770 & 770 & 770 & 770 & 770 \\
\hline
\end{tabular}

Here, Microcrystalline Cellulose (Avicel PH 101) was used as filler to keep the tablet weight of each batch constant. Lactose (fine) was used as channeling agent within the matrix of prepared tablets where Methocel K4M CR was used as release retarding agent. Magnesium stearate was used as lubricant to avoid capping and chipping problem of tablets. After wet mass preparation with purified water, half dried wet mass had been sieved through mesh no. 30 . Then LOD (loss on drying) of the granules was adjusted to $2.5 \%-3.0 \%$ at $95^{\circ} \mathrm{C}$. The drying temperature was maintained at $65^{\circ} \mathrm{C}$ to $70^{\circ} \mathrm{C}$. The granules then blended with Magnesium Stearate for two minutes only. Compressions of tablets were done on a 10 station single rotary machine using $17 \times 10$ $\mathrm{mm}$, caplet shaped punch.

Evaluation of tablets: The prepared matrix tablets were evaluated for hardness, weight variation, thickness, friability and drug content. Hardness of the tablets was tested using a strong-Cobb hardness tester (Tab-Machine, Mumbai). Friability of the tablets was determined in a Roche friabilator (Campbell Electronics, Mumbai). The thickness of the tablets was measured by vernier calipers. Weight variation test was performed according to the official method of
British Pharmacopoeia. Drug content determination for Niacin (Nicotinic Acid) was carried out according to USP30-NF25.

In-vitro Drug Release Studies: The formulated matrix tablets were subjected to the USP 24 dissolution apparatus type-I (paddle method) using $900 \mathrm{ml}$ of purified water as dissolution medium. The dissolution was performed at $100 \mathrm{rpm}$ and temperature was $37^{\circ} \mathrm{C} \pm 0.5^{\circ} \mathrm{C}$. At four predetermined time intervals $\left(1^{\text {st }}\right.$ hour, $4^{\text {th }}$ hour, $8^{\text {th }}$ hour and $20^{\text {th }}$ hour) over 20 hours period, 10 milliliters of samples were withdrawn, centrifuged and assayed spectrophotometerically (Shimadzu UV spectrophotometer, UV-1650 PC) at $263 \mathrm{~nm}$ after suitable dilution. After each sampling, equal volume $(10 \mathrm{ml})$ of fresh buffer solution with same temperature was replaced. All experiments were run as triplicate and averages were accounted.

Release kinetics: Based on In-vitro release studies, all data were fitted to various kinetic equations to find out the mechanism of drug release from the formulated matrix tablets. In this study four kinetic models as Zero order equation, First order equation, Square root of time equation or Higuchi equation and Korsmeyer-Peppas equation were used. 
Zero order equation: The equation (Eq. 1) assumes that the cumulative amount of drug release is directly related to time. The equation is as follows (Mutalik et al., 2007):

$$
C=K_{0} t
$$

Where, $K_{0}$ is the zero order rate constant expressed in unit concentration/time and $t$ is the time in hour.

First order equation: The release behavior of first order equation is expressed as log cumulative percentage of drug remaining verses time. The equation (Eq. 2) is as follows (Wagner, 1969):

Where,

$$
\log C=\log C_{0}-k t / 2.303
$$

$C=$ The amount of drug undissolved at $\mathrm{t}$

time

$C_{0}=$ Drug concentration at $t=0$

$k=$ Corresponding release rate constant.

Higuchi equation: The Higuchi release model describes the cumulative percentage of drug release verses square root of time. The equation (Eq. 3) is as follows (Higuchi, 1961):

$Q=K \sqrt{ } t \ldots \ldots \ldots \ldots$ (3)

Where, $Q=(100-C)$ the amount of drug dissolved at time $t . K$ is the constant reflecting the design variables of the system. Hence, drug release rate is proportional to the reciprocal of the square root of time.

Korsmeyer-Peppas equation: Korsmeyer et al developed a simple, semi-empirical model, relating exponentially the drug release to the elapsed time (Korsmeyers et al., 1983). The equation (Eq. 4) is as follows:

$$
Q / Q_{0}=K t^{n}
$$

Where,

$Q / Q_{0}=$ The fraction of drug released at time $t$

$k=$ Constant comprising the structural geometric characteristics

$n=$ The diffusion exponent that depends on the release mechanism If $n \leq 0.5$, the release mechanism follows a Fickian diffusion, and if $0.5<n<1$, the release follows a non-Fickian diffusion or anomalous transport. The drug release follows zero order drug release and case-II transport if $n=1$. But when $n>1$, then the release mechanism is super case-II transport. This model is used in the polymeric dosage form when the release mechanism is unknown or more than one release phenomena is present in the preparation (Peppas, 1985).

\section{RESULTS AND DISCUSSION}

Physical properties: The results of physical parameters (i.e., weight, hardness, thickness and friability) and drug content of the prepared matrix tablets were shown in Table 2. The hardness of tablets ranged from $10.1 \pm 0.02 \mathrm{~kg} / \mathrm{cm}^{2}$ to $10.5 \pm 0.03$ $\mathrm{kg} / \mathrm{cm}^{2}$ and friability ranged from $0.09 \%$ to $0.15 \%$. The weight variations of prepared tablets found were complied with the pharmacopoeial specifications. The drug content of every formulation was found about to $100 \%$ of labeled content. All these results indicate that the granules possessed satisfactory flow properties, compressibility and drug content.

Table 2: Physical properties of the Niacin extended release matrix tablet of the proposed formulations (F-1 to F-6)

\begin{tabular}{|c|c|c|c|c|c|}
\hline Formulation & $\begin{array}{c}\text { Hardness } \\
\mathbf{K g} / \mathbf{c m}^{\mathbf{2}} \\
\mathbf{\pm} \mathbf{S D}(\mathbf{n}=\mathbf{5})\end{array}$ & $\begin{array}{c}\text { Thickness mm } \\
\mathbf{\pm} \mathbf{S D}(\mathbf{n}=\mathbf{5})\end{array}$ & Friability \% & $\begin{array}{c}\text { Drug content } \\
\mathbf{( \% )} \\
\mathbf{\pm} \text { SD (n= 5) }\end{array}$ & $\begin{array}{c}\text { Weight mg } \\
\mathbf{\pm} \text { SD } \\
(\mathbf{n}=\mathbf{2 0})\end{array}$ \\
\hline F-1 & $10.1 \pm 0.21$ & $4.15 \pm 0.02$ & 0.15 & $99.98 \pm 0.57$ & $780.2 \pm 1.61$ \\
\hline F-2 & $10.1 \pm 0.25$ & $4.17 \pm 0.03$ & 0.13 & $99.48 \pm 0.78$ & $780.1 \pm 1.52$ \\
\hline F-3 & $10.5 \pm 0.30$ & $4.21 \pm 0.01$ & 0.10 & $99.52 \pm 0.94$ & $779.9 \pm 1.55$ \\
\hline F-4 & $10.2 \pm 0.23$ & $4.23 \pm 0.04$ & 0.11 & $98.09 \pm 1.12$ & $780.5 \pm 1.62$ \\
\hline F-5 & $10.3 \pm 0.27$ & $4.14 \pm 0.02$ & 0.11 & $99.85 \pm 0.42$ & $780.5 \pm 1.29$ \\
\hline F-6 & $10.5 \pm 0.31$ & $4.13 \pm 0.01$ & 0.09 & $99.05 \pm 0.67$ & $780.1 \pm 1.37$ \\
\hline
\end{tabular}

SD-Standard deviation, $\mathrm{n}$-Number of replicates

In-vitro release kinetics: Table 3 shows data analysis of release profile according to different kinetic models. The drug releases from tablets were found slow and extended over 20 hours. The basic characteristics of HPMC polymer is, they undergo rapid hydration and chain relaxation to form viscose gelatinous layer (gel layer) when exposed to aqueous medium. This gel layer acts as the barrier to release of drug and make the release prolong. Failure to generate a uniform and coherent gel may cause dose 
dumping. Lactose (fine) acts as a channeling agent for the penetration of coarse aqueous medium. It makes a network of channel throughout the gel layer to diffuse out the active into the dissolution medium.

The data from Table 3 shows that although some of formulations (F-2, F-5 and F-6) follow zero-order kinetics while most of the formulations (F-1, F-2, F-4, F-5 and F-6) follow first order kinetics. The drug release from all the Formulations (except F-3) can be best described through Higuchi Kinetic model. Because when the data were plotted according to the Higuchi square root equation, the formulations (except F-3) showed a fair linearity, with regression values ranging from 0.914 to 0.987 . From the data of
Table 3, it is clear that F-5 is best fitted in terms of Higuchi model $\left(R^{2}=0.987\right)$ and also in term of Korsmeyer-Peppas model where its drug release mechanism is an anomalous (Non-Fickian) diffusion process $(\mathrm{n}=0.714)$. About $15.58 \%$ of HPMC (Methocel K4M CR) polymer in this formula (F-5) alone is sufficient to control drug release. Amount of channeling agent like Lactose also plays a vital role to make gel layer and also diffuse out the drug through the HPMC matrix. It is found that $10.65 \%$ Lactose of F-5 formula produces appropriate amount of pores within the matrix to penetrate the dissolution medium to swell and relax the polymer chain of HPMC.

Table 3: Release rate parameters [ $Y$-equation $(Y=a X+b)$ and Correlation co-efficient $\left(R^{2}\right)$ and release exponent $(n)$ ] for release data after fitting of the whole release profiles of Niacin ER matrix tablets of different formulations from four different kinetic models

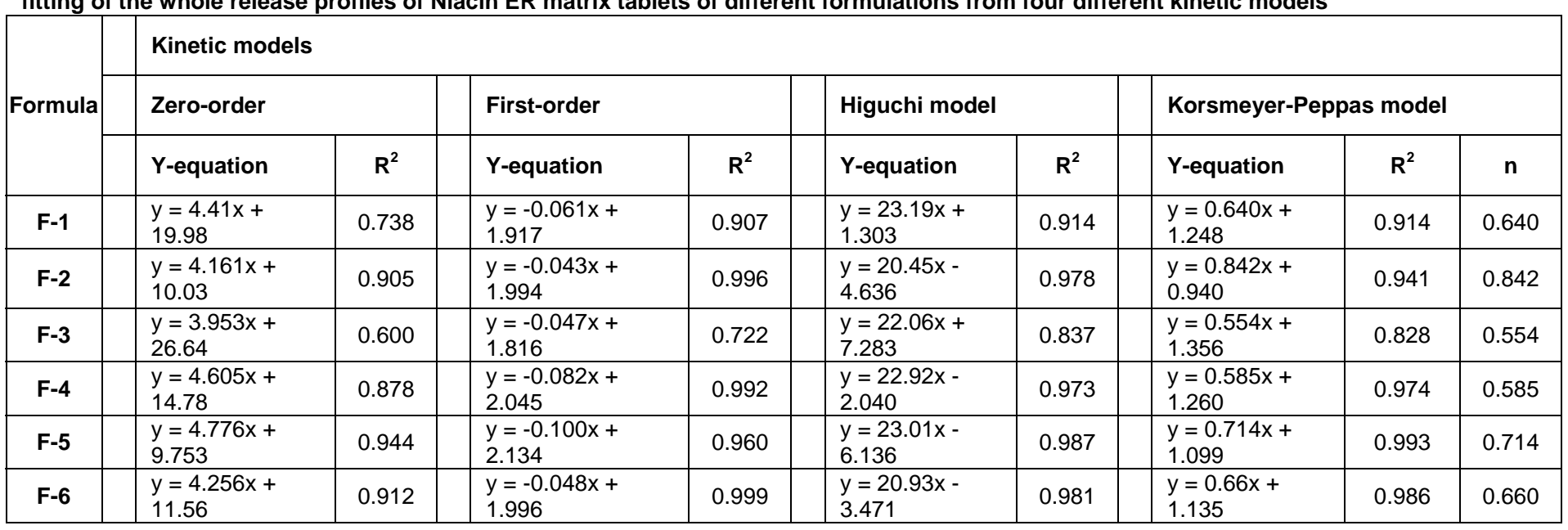


Am. J. Sci. Ind. Res., 2010, 1(3): 558-564

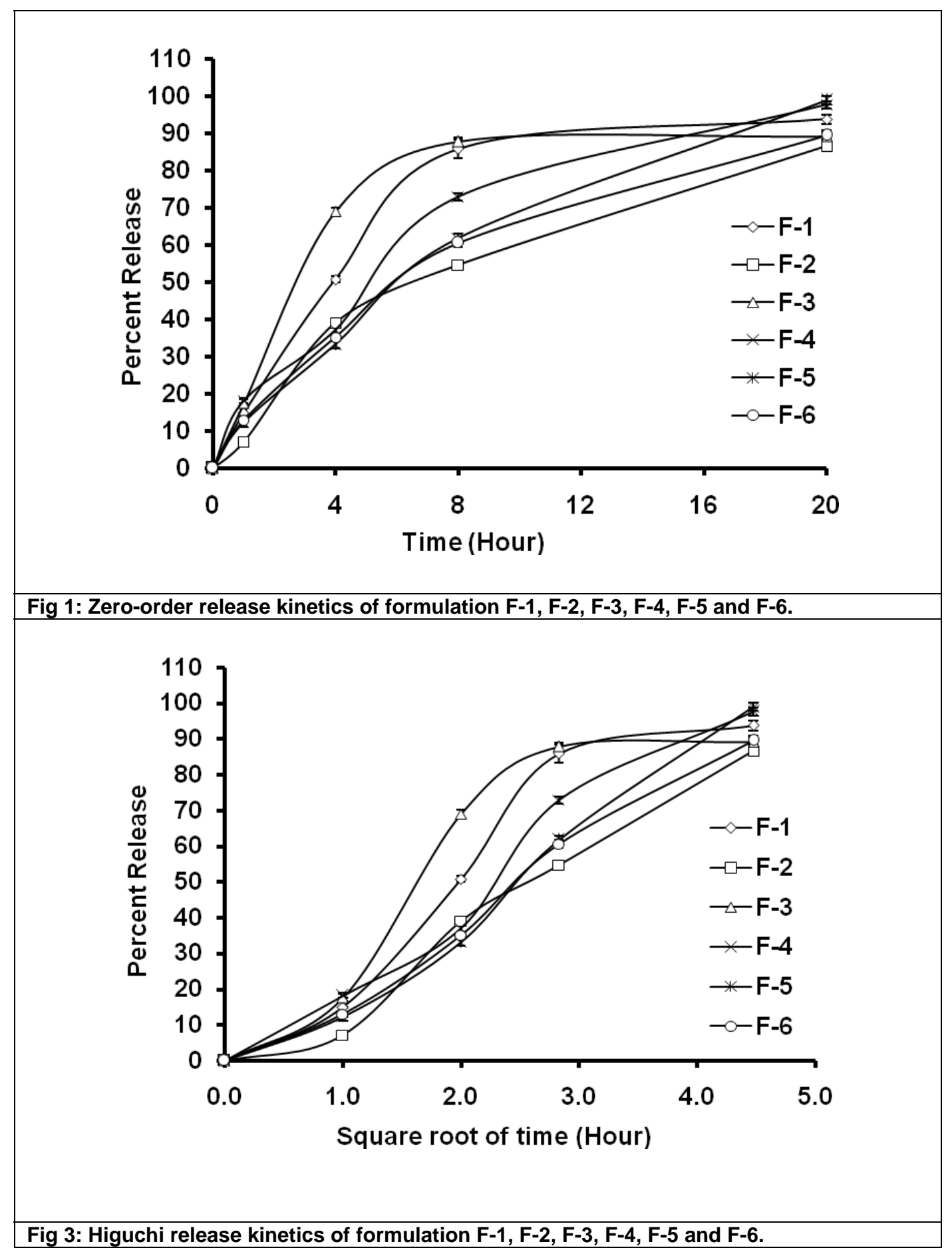


Am. J. Sci. Ind. Res., 2010, 1(3): 558-564

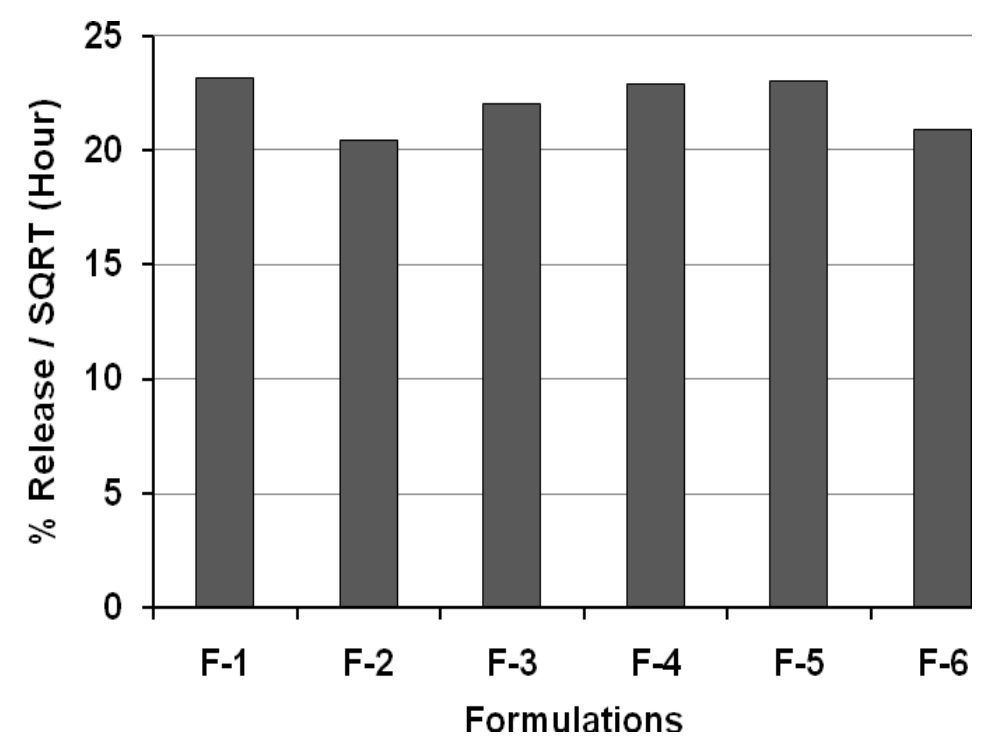

Fig 5: Release rate of Niacin from different formulations F-1, F-2, F-3, F-4, F-5 and F-6.

\section{CONCLUSION:}

Niacin is a safe and effective therapeutic agent for the management of patients with dyslipidemias. The most therapeutic benefit from Niacin therapy with minimal adverse effects is optimally attained by Niacin ER, which has an efficacy profile similar to Niacin IR, a reduced incidence of flushing compared with Niacin IR, and no increased risk of hepatotoxicity as is seen with Niacin SR. In this regards, the best proposed formulation with respective percentage of HPMC may be used for the development of Niacin ER matrix tablet which affects all aspects of the lipid profile, and should be considered an important therapeutic option for patients with dyslipidemias.

\section{REFERENCES}

Canner PL, Berge KG, Wenger NK, et al, for the Coronary Drug Project Research Group. Fifteen year mortality in Coronary Drug Project patients: long-term benefit with niacin. J Am Coll Cardiol. 1986; 8: 1245-1255.

Grundy SM, Mok HY, Zech L, Berman M. Influence of nicotinic acid on metabolism of cholesterol and triglycerides in man. J Lipid Res. 1981; 22: 24-36.

Higuchi T. Rate of release of medicaments from ointment bases containing drugs in suspension. J. Pharm. Sci, 1961: 50: 847-875.
Knopp $\mathrm{RH}$, Alagona $\mathrm{P}$, Davidson $\mathrm{M}$, et al. Equivalent efficacy of a time-release form of niacin (Niaspan) given once-a-night versus plain niacin in the management of hyperlipidemia. Metabolism. 1998; 47:1097-1104.

Knopp RH, Ginsberg J, Albers JJ, et al. Contrasting effects of unmodified and time-release forms of niacin on lipoproteins in hyperlipidemic subjects: clues to mechanism of action of niacin. Metabolism. 1985; 34: 642-650.

Knopp RH. Evaluating niacin in its various forms. Am J Cardiol. 2000; 86(suppl):51L-56L.

Korsmeyers RW, Gumy R, Doelker EM, Buri P, Peppas NA. Mechanism of solute release from porous hydrophilic polymers, Int J Pharm, 1983: 15: 25-35.

McKenney JM, Proctor JD, Harris S, Chinchili VM. A comparison of the efficacy and toxic effects of sustained- vs immediate-release niacin in hypercholesterolemic patients. JAMA. 1994; 271:672677.

Mutalik S, Naha A., Usha AN, Ranjith. AK, Preparation, In vitro, preclinical and clinical evaluations of once daily sustained release tablets of aceclofenac. Arch Pharm Res. 2007; Vol 30, No 2, 222-234. 
Am. J. Sci. Ind. Res., 2010, 1(3): 558-564

Peppas NA. Analysis of Fickian and non-Fickian drug release from polymers. Pharm Acta. Hel., 1985: 60:110 -111.

The Coronary Drug Project Research Group. Clofibrate and niacin in coronary heart disease. JAMA. 1975; 231:360-381.
Wagner, J.G. Interpretation of percent dissolved-time plots derived from in vitro testing of conventional tablets and capsules. J. Pharm. Sci. 1969; 58, 1253-1257. 